\title{
MicroRNA-29b inhibits peritoneal fibrosis in a mouse model of peritoneal dialysis
}

\author{
Jian-Wen Yu ${ }^{1,2,4}$, Wen-Juan Duan ${ }^{1,2,4}$, Xiao-Ru Huang ${ }^{2,3}$, Xiao-Ming Meng ${ }^{2}$, Xue-Qing Yu ${ }^{1}$ and Hui-Yao Lan 2,3
}

TGF- $\beta /$ Smad3 signaling plays a pivotal role in the pathogenesis of peritoneal fibrosis associated with peritoneal dialysis (PD). MicroRNA-29 (miR-29) is known as a potent downstream inhibitor of TGF- $\beta /$ Smad3 in renal fibrosis. In this study, we examined the therapeutic potential for miR-29b on PD-related peritoneal fibrosis in a mouse model of PD induced by daily infusion of $4.25 \%$ dextrose-containing PD fluid (PDF). MiR-29b-expressing plasmid was delivered into the peritoneum via an ultrasound-microbubble-mediated system before and at day 14 after PDF. We found that mice on PD developed peritoneal fibrosis with impaired peritoneal function, which was associated with a loss of miR-29b. In contrast, overexpression of miR-29b before the PDF infusion showed a protective effect on peritoneal fibrosis including EMT and prevented peritoneal dysfunction. Moreover, delayed miR-29b treatment until peritoneal fibrosis was established at day 14 also halted the progression of peritoneal fibrosis at day 28. Further studies identified that blockade of the Sp1-TGF- $\beta /$ Smad3 pathway may be a mechanism by which miR-29b inhibited peritoneal fibrosis. In conclusion, treatment with miR-29b may represent a novel and effective therapy for PD-associated peritoneal fibrosis.

Laboratory Investigation (2014) 94, 978-990; doi:10.1038/labinvest.2014.91; published online 21 July 2014

Peritoneal dialysis (PD) is a well-accepted therapeutic strategy for patients with end-stage renal disease. However, long-term PD is limited because of peritoneal fibrosis in response to chronic infusion of the bioincompatible $\mathrm{PD}$ fluid (PDF). After long-term exposure to PDF, the peritoneum undergoes histological changes including a loss of mesothelial cell monolayer accompanied by excessive deposition of extracellular matrix and angiogenesis. ${ }^{1,2}$ Although targeting fibrosis and/or angiogenesis with antisense or peptide reagents have been shown to inhibit peritoneal fibrosis, ${ }^{3-5}$ specific and effective anti-fibrosis therapies in PD remain to be explored.

Mounting evidence shows that TGF- $\beta 1$ plays a pivotal role in the development of PD-related peritoneal fibrosis. Persistent elevation of TGF- $\beta 1$ in effluents from PD patients with frequent peritonitis is associated with increased risk for peritoneal fibrosis. ${ }^{6}$ Overexpression of TGF- $\beta 1$ using adenovirusmediated gene transfer in rat peritoneum recapitulates the structural and functional changes observed in patients with PD. ${ }^{7,8}$ In contrast, inhibition of TGF- $\beta 1$ ameliorates the damage to the peritoneum in different animal models. ${ }^{9}$ It is well established that Smads are key intracellular effectors of TGF- $\beta$ signaling and Smad3, but not Smad2, is an essential mediator in TGF- $\beta 1$-induced renal fibrosis. ${ }^{10,11}$ Similarly, it has been reported that deletion of Smad3 protects against peritoneal fibrosis. ${ }^{12}$ However, as TGF- $\beta / \mathrm{Smad} 3$ is indispensable in maintaining homeostasis of the immune system, ${ }^{13,14}$ it raises concerns about long-term consequences to treat fibrosis by blocking the general effects of this pathway. Thus, targeting the TGF- $\beta /$ Smad3- related gene(s) associated with fibrosis may represent a better therapy approach for treatment of peritoneal fibrosis.

MicroRNAs (miRNAs) are small regulatory RNAs that may have important roles in a wide range of pathophysiological processes. Recent findings indicated that the miR-29 family is one of the best characterized miRNAs related to TGF- $\beta 1$-mediated fibrosis. ${ }^{15}$ Indeed, miR-29 is predicted to target a multitude of extracellular matrix genes, such as ELN, FBN1, COL1A1, COL1A2, and COL3A1. ${ }^{16}$ Thus, loss of miR-29 contributes to fibrosis in the heart, liver, and lung diseases. ${ }^{16-19}$ We and other investigators also identify that miR-29b is a target gene of TGF- $\beta /$ Smad 3 and overexpression

\footnotetext{
'Department of Nephrology, The First Affiliated Hospital, Sun Yat-Sen University, Guangzhou, China; ${ }^{2}$ Li Ka Shing Institute of Health Sciences and Department of Medicine \& Therapeutics, The Chinese University of Hong Kong, Hong Kong, China and ${ }^{3}$ Shenzhen Research Institute, The Chinese University of Hong Kong, Shenzhen, China

Correspondence: Professor X-Q Yu, MD, PhD, Department of Nephrology, The First Affiliated Hospital, Sun Yat-Sen University, Guangzhou, China or Professor H-Y Lan, MD, PhD, Department of Medicine and Therapeutics, and Li Ka Shing Institute of Health Sciences, The Chinese University of Hong Kong, Hong Kong, China. E-mail: yuxq@mail.sysu.edu.cn or hylan@cuhk.edu.hk

${ }^{4}$ These authors contributed equally to this work.

Received 26 February 2014; revised 21 May 2014; accepted 2 June 2014
} 
of miR-29b is capable of inhibiting TGF- $\beta /$ Smad3-mediated fibrosis in the heart, lung, and kidney diseases. ${ }^{17,20-23}$ However, it remains unknown whether miR-29 has therapeutic effect on peritoneal fibrosis. Therefore, the present study examined if miR-29 has therapeutic potential for peritoneal fibrosis in a mouse model of PD.

\section{MATERIALS AND METHODS}

Gene Transfer of Inducible MiR-29b into the Peritoneum via an Ultrasound-Microbubble-Mediated System

A Dox-regulated miR-29b-expressing plasmid pTRE $_{2}$-miR-29b was generated as previously reported. ${ }^{17}$ The empty vector (EV) $\mathrm{pTRE}_{2}$-hygro was used as a control. To achieve Dox-induced transgene expression, $\mathrm{pTRE}_{2}$-miR-29b/pTRE -hygro and $^{-}$ pEFpuro-p-Tet-on were co-transfected into the abdominal cavity via an ultrasound-microbubble delivery system as described previously with some modifications. ${ }^{24,25}$ Briefly, a mixture of plasmids ( $\mathrm{pTRE}_{2}$-miR-29b or $\mathrm{pTRE}_{2}$-hygro EV) and Sonovue (Bracco Diagnostics, Princeton, NJ, USA) was prepared with 1:1 vol/vol ratio. Then the mixed solution containing $200 \mu \mathrm{g}$ of designated plasmids in total volume of $1 \mathrm{ml}$ was immediately injected into the peritoneal cavity. The ultrasound probe (Sonitron 2000; Rich-Mar Corp., Inola, OK, USA) was placed vertically to the anterior abdominal surface and moved over the entire anterior abdominal wall to allow the ultrasound beam to reach the whole peritoneum. The ultrasound treatment was performed at $1 \mathrm{MHz}$ input frequency and $2 \mathrm{~W} / \mathrm{cm}^{2}$ output intensity for $5 \mathrm{~min}$. To induce miR-29b transgene expression, a dose of Dox $(300 \mu \mathrm{l})$ with a concentration of $200 \mu \mathrm{g} / \mathrm{ml}$ was administered into the peritoneal cavity just after ultrasound exposure and followed by $200 \mu \mathrm{g} / \mathrm{ml}$ of Dox in the daily drinking water over the entire study period. The same protocol was applied to mice treated with either control or miR-29b plasmids.

\section{Animal Experimental Protocol}

Male C57BL/6J mice (8-10-weeks old) purchased from Laboratory Animal Services Centre, the Chinese University of Hong Kong, were used in this study. A mouse model of peritoneal fibrosis was induced by daily intraperitoneal injection of $3 \mathrm{ml}$ of $4.25 \%$ dextrose PDF (Baxter HealthCare, Deerfield, IL, USA) as previously described with some modifications. ${ }^{24}$ We first performed pilot studies to determine miR-29b transfection rate and transgene expression in the peritoneal tissues. Groups of three to four mice treated with ultrasound-mediated $\mathrm{pTRE}_{2}$-miR-29b plasmids were killed at $1,2,3$, and 4 weeks after ultrasound treatment. Consistent with our previous finding in heart and kidney diseases, ${ }^{17,21}$ a significant higher level of miR-29b expression was observed at the first 2 weeks after miR-29b gene transfer. Thus, a second ultrasound-microbubble-miR-29b treatment was given at day 14 in the experiment over 28 days.

Two experiments were conducted in this study. First, to examine the protective role of miR-29b in peritoneal fibrosis, groups of six mice were treated with ultrasound-mediated
pre-miR-29b or control EVs gene transfer (termed day 0), followed immediately by daily PDF infusion. All mice were given the second ultrasound-microbubble-miR-29b or control treatment at day 14 and killed at day 28. Second, to determine the therapeutic potential of miR-29b in peritoneal fibrosis, groups of six mice were treated with pre-miR-29b or control plasmids at day 14 after PD and killed at day 28. In addition, groups of six mice under PD but without treatment were killed at day 14 and day 28 as untreated-disease controls. All experimental procedures were approved by the Animal Experimentation Ethics Committee at the Chinese University of Hong Kong.

Peritoneal tissues including the anterior abdominal wall and omentum were collected, fixed in methyl Carnoy's for histology and immunohistochemistry or snap-frozen for RNA and protein extraction. For in situ hybridization, the anterior abdominal wall was fixed in $4 \%$ paraformaldehyde solution.

\section{Analysis of Peritoneal Permeability}

Modified peritoneal equilibration tests were performed to determine the peritoneal permeability before the mice were killed. After intraperitoneal injection of $3 \mathrm{ml}$ of $4.25 \%$ dextrose of PDF, dialysate and blood samples were collected at 0 and $120 \mathrm{~min}$ of dwell time. The concentrations of glucose and BUN in the dialysate and plasma were measured using commercial kits (Stanbio Laboratory, Boerne, TX, USA). The peritoneal permeability was determined by the absorption of glucose from the dialysate $(\mathrm{D} / \mathrm{D} 0)$ and the dialysateto-plasma $(\mathrm{D} / \mathrm{P})$ ratio of blood urea nitrogen.

\section{RNA Extraction, Quantitative Real-Time PCR, and In Situ Hybridization}

Total RNA was extracted from peritoneal tissues using Tri-Reagent (Invitrogen, Carlsbad, CA, USA) according to the manufacturer's protocol. Real-time PCR for miR-29b was performed using the TaqMan microRNA assay kit (Applied Biosystems, Foster City, CA, USA) and Bio-Rad iQ SYBR Green supermix with Opticon2 (Bio-Rad, Hercules, CA, USA). The primers used in this study including mouse miR-29b, U6, collagen I, $\alpha$-SMA, $\beta$-actin, and TGF- $\beta 1$ were described previously. ${ }^{20-22}$ The housekeeping genes U6 and $\beta$-actin were used as internal controls. The ratio for the mRNA of interest normalized with internal controls was expressed as mean \pm s.e.m.

For in situ hybridization, specific LNA-digoxigenin-labeled mmu-miR-29b probe $\left(5^{\prime}\right.$-AACACTGATTTCAAATGGTG CTA- $\left.3^{\prime}\right)$ or scramble control probe $\left(5^{\prime}\right.$-GTGTAACAC GT CTATACGCCCA- $3^{\prime}$ ) were purchased from Exiqon (Vedbaek, Denmark). In situ hybridization of miR-29b was performed in paraformaldehyde-fixed, paraffin-embedded peritoneal sections as described previously. ${ }^{20-22}$

\section{Histology, Immunohistochemistry, and Two-Color Immunofluorescence}

All histological and immunohistochemical analyses were performed on methyl Carnoy's-fixed, paraffin-embedded 
sections. Deparaffinized sections of the anterior abdominal wall were stained with Masson's trichrome stain kit (ScyTek Laboratories, Logan, UT, USA) to quantify fibrosis. The thickness of the submesothelial collagenous zone was determined under microscope in coded slides using a metric ocular and was expressed as the mean of 10 independent measurements for each mouse section. Immunohistochemistry was carried out using a microwave-based antigen retrieval technique. ${ }^{26}$ The primary antibodies used in this study included collagen I (SouthernBiotech, Birmingham, AL, USA), $\alpha$-SMA (Sigma), CD31, VEGF, p-Smad2/3, and TGF$\beta 1$ (Santa Cruz Biotechnology, Dallas, TX, USA). CD31positive blood vessels in the omentum and $\mathrm{p}$-Smad2/3-positve cells in the anterior abdominal wall were counted under high power fields by means of a graticule fitted in the eyepiece of the microscope and expressed as positive vessels $/ \mathrm{mm}^{2}$ or positive cells/high power field. Percentages of VEGF-positive area in the omentum were quantified by the Image-Pro plus 7.0 software (Media Cybernetics, Bethesda, MD, USA) as reported previously. ${ }^{20-22}$ TGF- $\beta 1$ expression in the anterior abdominal wall was semiquantitatively analyzed based on positive staining intensity: $0=$ no staining, $1=$ mild, $2=$ moderate, $3=$ strong staining. All quantitative analyses were performed in coded slides and 10 consecutive high power fields were assessed for each mouse section. A mean value from 10 independent measurements was calculated for statistical analysis.

To examine epithelial-to-mesenchymal transition (EMT) during peritoneal fibrosis, two-color immunofluorescence was performed on the snap-frozen peritoneal tissue sections. Sections were incubated with Cy3-conjugated antibody against $\alpha$-SMA(Sigma) and a rabbit anti- mouse cytokeratin (Santa Cruz Biotechnology) for overnight at $4{ }^{\circ} \mathrm{C}$, followed by the goat anti-rabbit FITC-conjugated IgG (Dako, Glostrup, Denmark) for $1 \mathrm{~h}$. Nuclei were counterstained with DAPI. The EMT cells were identified by co-expressing $\alpha$-SMA (red) and cytokeratin (green) antigens under fluorescent microscope (Axioplan2 imaging, Carl Zeiss, Oberkoche, Germany).

\section{Western Blot Analysis}

Protein from peritoneal tissues was extracted as previously reported. ${ }^{20-22}$ The primary antibodies used in this study included collagen I (SouthernBiotech), E-cadherin (BD Transduction Laboratories, San Jose, CA, USA), $\alpha$-SMA (Dako), Sp1, and $\beta$-actin (Santa Cruz Biotechnology). The signals were detected by the LiCor/Odyssey infrared image system (LI-COR Biosciences, Lincoln, NE, USA) and quantified by Image $\mathrm{J}$ software (National Institutes of Health). The ratio for the protein of interest was normalized against $\beta$-actin and expressed as mean \pm s.e.m.

\section{Statistical Analysis}

Data from this study were expressed as mean \pm s.e.m. Statistical analyses were performed using one-way analysis of variance, followed by Newman-Keuls Post Test from the GraphPad Prism 5.0 software (San Diego, CA, USA).

\section{RESULTS \\ MiR-29b Gene Transfer Prevents Peritoneal Fibrosis in a Mouse Model of Peritoneal Dialysis}

It is known that within the miR-29 family, miR-29a is co-expressed with miR-29b-1 and miR-29c is co-expressed with miR-29b-2. ${ }^{16}$ Thus, miR-29b is a representative miRNA of the miR-29 family and was used in this study. We first examined the efficiency of ultrasound-microbubble-mediated miR-29b transgene expression in the peritoneum. By in situ hybridization, we detected that moderate miR-29b was constitutively expressed by peritoneal mesothelial cells and cells in the submesothelial area and muscles in the anterior abdominal wall in normal mice, but this was largely decreased in the fibrotic peritoneum after PDF for 28 days (Figure 1a). In contrast, PD mice that were given miR-29b gene transfer exhibited a strong expression of miR-29b in the peritoneal tissues including mesothelial cells and fibroblastlike cells in the submesothelial area (Figure 1a). Quantitative real-time PCR analysis of the omentum tissues confirmed this finding (Figure 1c).

Next we examined the preventive effect of miR-29b on peritoneal fibrosis and functional injury in response to the chronic PDF infusion. Masson's trichrome staining revealed that compared with normal mice, mice on PD receiving no treatment or control EVs showed a typical feature of peritoneal fibrosis, including loss of the mesothelial cell monolayer and the thickening of submesothelial compact zone within the anterior abdominal wall (Figure $1 \mathrm{~b}$ and $\mathrm{d}$ ). These histological changes were associated with the increased peritoneal permeability as determined by peritoneal equilibration tests (Figure 1e and f). In contrast, miR-29b transfer prevented the peritoneal thickening and excessive matrix deposition as well as peritoneal dysfunction (Figure 1). These protective effects on peritoneal fibrosis were further demonstrated by the ability of overexpressing miR-29b to inhibit collagen I expression at both protein and mRNA levels (Figure $2 \mathrm{a}-\mathrm{c}$ ) and to block EMT by restoration of E-cadherin whereas significantly inhibiting $\alpha$-SMA expression and cytokeratin $+\alpha$-SMA + cells in the thickened peritoneum (Figure $2 \mathrm{~d}-\mathrm{g}$ ). Furthermore, we also found that overexpression of miR-29b largely inhibited peritoneal angiogenesis by significantly reducing CD31-positive blood vessels and expression of VEGF in the fibrotic peritoneal tissues (Figure 3).

\section{MiR-29b Gene Transfer Retards Progressive Peritoneal Fibrosis in a Mouse Model of Established PD}

We then examined whether miR-29b has therapeutic effect on peritoneal fibrosis in a mouse model of established PD. Realtime PCR showed that a daily injection of PDF for 14 days resulted in the reduction of miR-29b expression, becoming almost lost by day 28 (Figure 4a). Reduced peritoneal miR-29b expression at day 14 was associated with the development of a 


\section{PD}

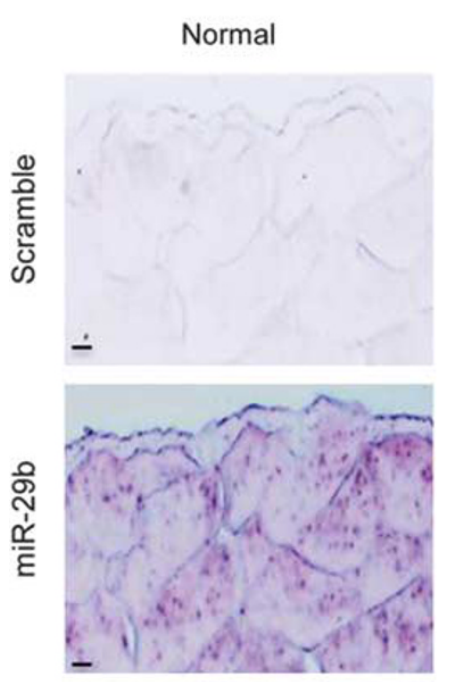

Untreated (UT)
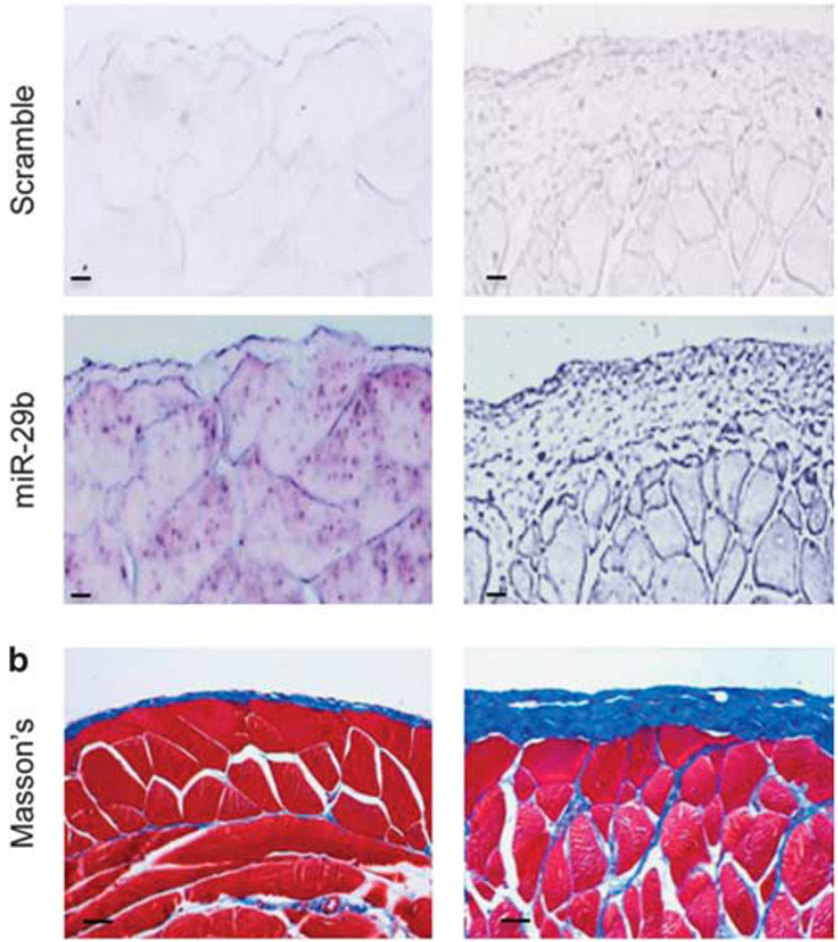

C

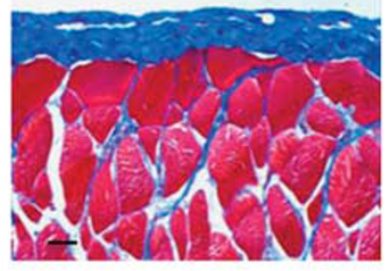

miR-29b

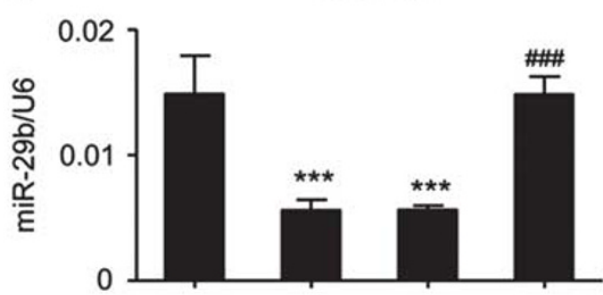

e

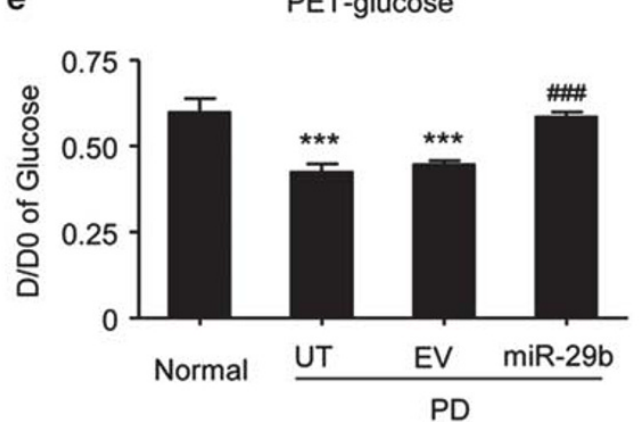

EV
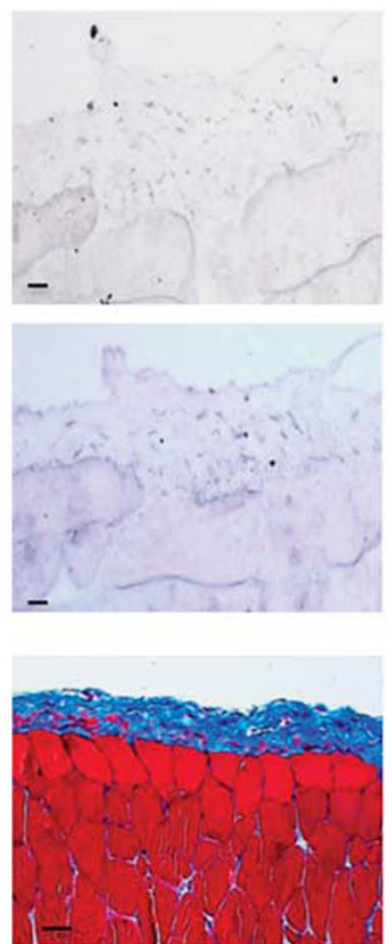

miR-29b
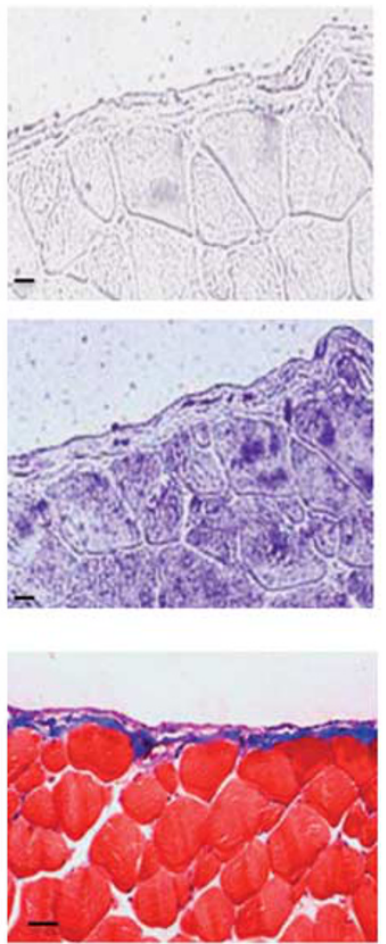

d

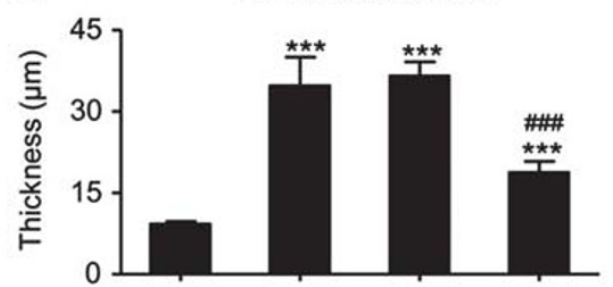

PET-BUN

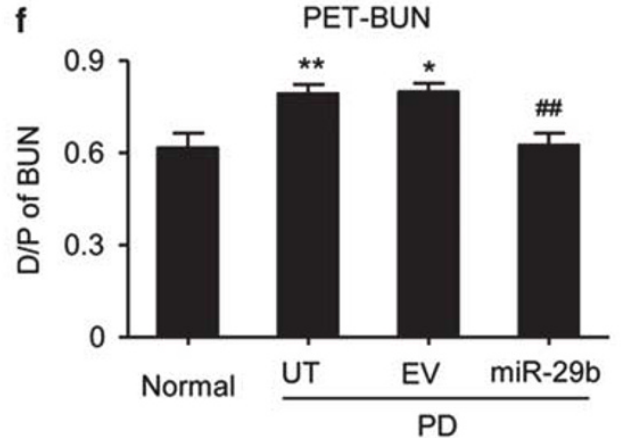

Figure 1 Ultrasound-microbubble-mediated miR-29b gene transfer prevents peritoneal fibrosis and functional injury in a mouse model of PD. (a) In situ hybridization shows that a moderate amount of miR-29b is expressed by mesothelial cells, submesothelial cells, and muscle cells in normal mice, which is lost in untreated (UT) mice or mice receiving the control empty vector (EV) at day 28 after PD, but restored by miR-29b transfer (miR-29b).

(b) Masson's trichrome staining of anterior abdominal walls. (c) Real-time analysis of miR-29b expression in the peritoneal tissues. (d) Thickness of the peritoneal membrane. (e and $\mathbf{f}$ ) PET-glucose and PET-BUN analysis of peritoneal function. Each bar represents mean \pm s.e.m.

for at least five mice. ${ }^{*} P<0.05,{ }^{* *} P<0.01,{ }^{* * *} P<0.001$ versus normal controls; ${ }^{\# \#} P<0.01,{ }^{\# \# \#} P<0.001$ versus $\mathrm{EV}$ control. Scale bar $=50 \mu \mathrm{M}$. 


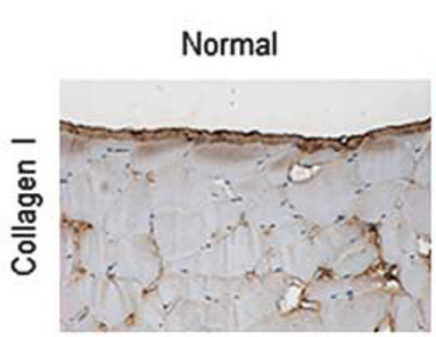

b

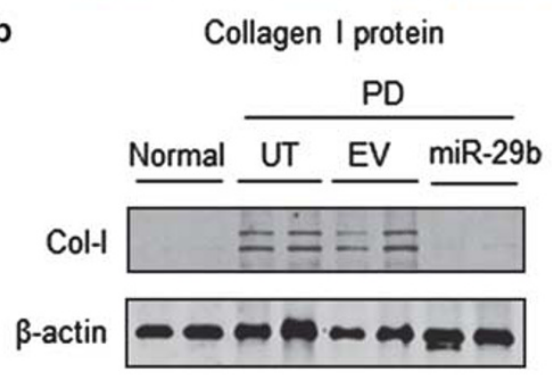

d

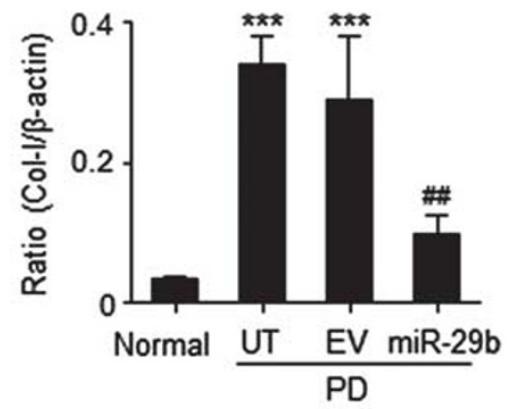

PD
EV
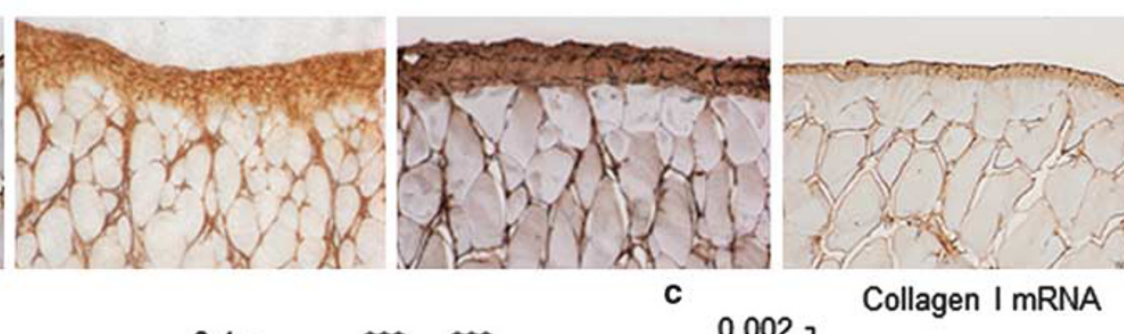

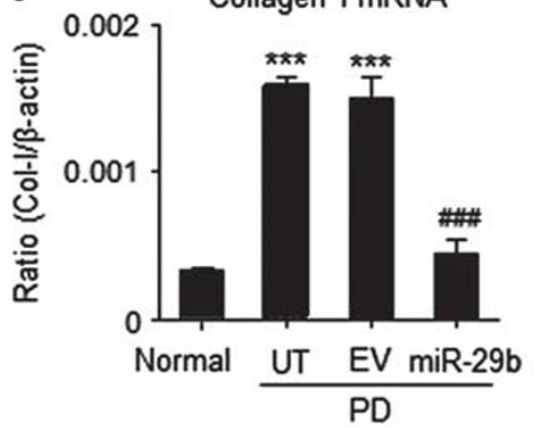

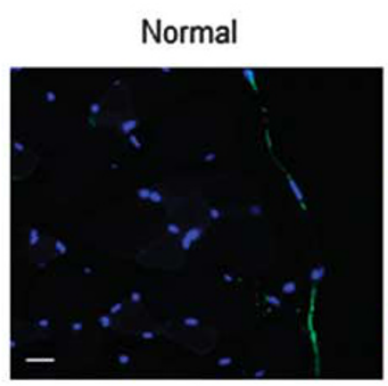

e E-Cadherin protein
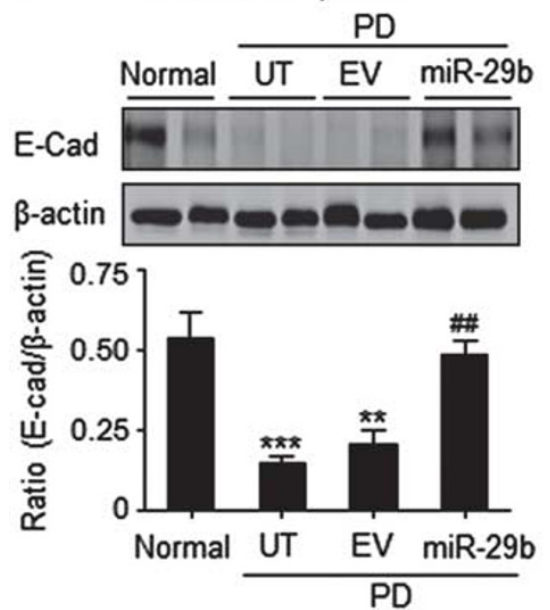
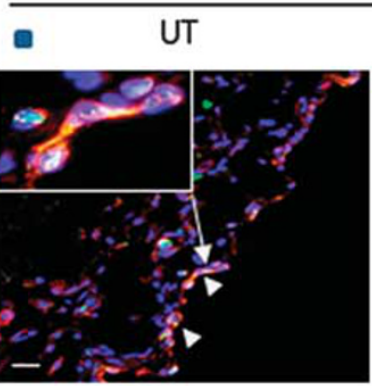

f
EV

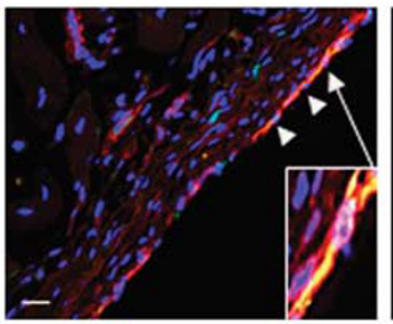

$\alpha$-SMA protein g
miR-29b

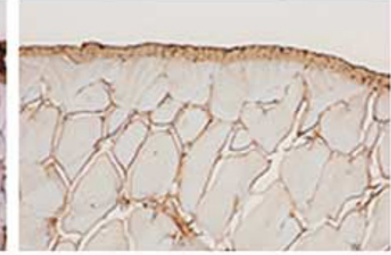

Collagen I mRNA

Normal UT EV miR-29b
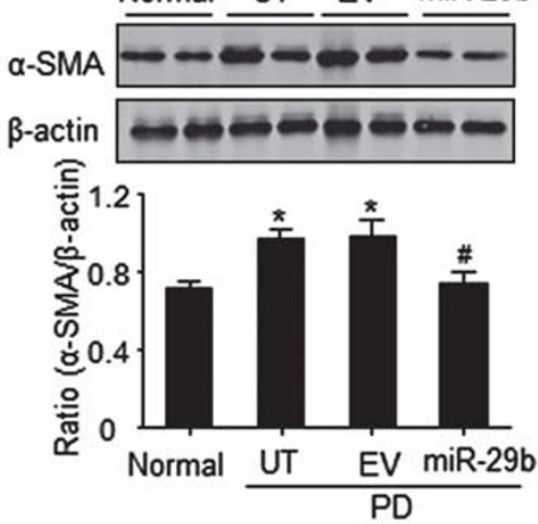

-SMA mRNA

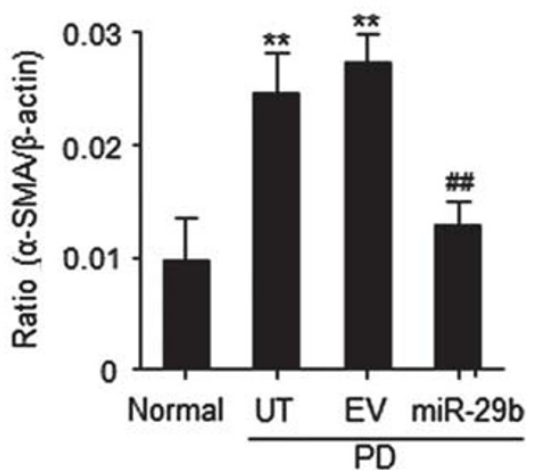

Figure 2 Pre-miR-29b treatment prevents collagen I deposition, $\alpha$-SMA + myofibroblast accumulation, and epithelial-to-mesenchymal transition (EMT) in a mouse model of PD. (a) Immunostaining of collagen I deposition in the submesothelial area of the anterior abdominal wall. (b) Western blot analysis of peritoneal collagen I expression. (c) Real-time PCR analysis of peritoneal collagen I mRNA expression. (d) Two-color immunofluorescence of anterior abdominal wall sections. Note that EMT cells (arrowheads) are identified in both untreated (UT) and control empty vector (EV)-treated fibrotic peritoneal tissues by being dual positive for cytokeratin (green) and $\alpha$-SMA (red), which is further illustrated in the inserted picture. Treatment of miR-29b virtually blocked EMT. (e and f) Western blot analysis of peritoneal E-Cadherin and $\alpha$-SMA expression. (g) Real-time PCR analysis of peritoneal $\alpha$-SMA mRNA expression. Each bar represents mean \pm s.e.m. for at least five mice. ${ }^{*} P<0.05,{ }^{*} P<0.01,{ }^{* * *} P<0.001$ versus normal controls; ${ }^{*} P<0.05$, ${ }^{\# \#} P<0.01,{ }^{\# \# \#} P<0.001$ versus $\mathrm{PD}$ mice receiving control EV. Scale bar $=50 \mu \mathrm{M}$. 

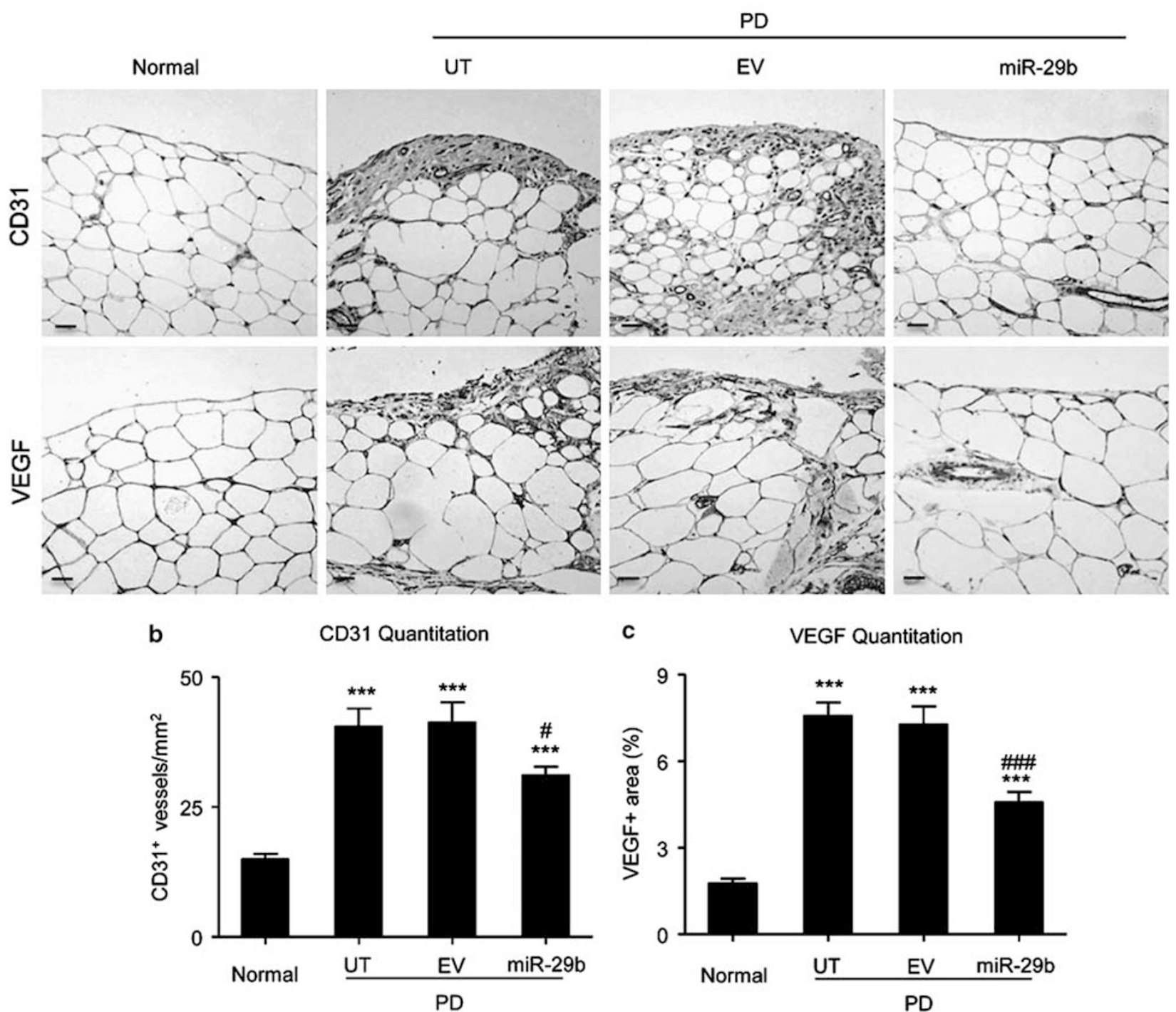

b

C

VEGF Quantitation

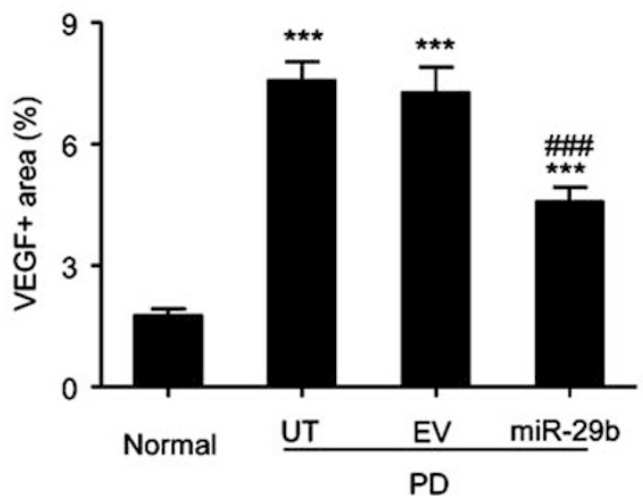

Figure 3 Pre-miR-29b treatment prevents peritoneal angiogenesis in a mouse model of PD. (a) Immunostaining of CD31 + vessels and VEGF + cells in the omentum. (b) Quantitative analysis of CD31-positive blood vessels. (c) Quantitative analysis of VEGF expression. Each bar represents mean \pm s.e.m. for at least five mice. ${ }^{* * *} P<0.001$ versus normal controls; ${ }^{\#} P<0.05$, ${ }^{\# \# \#} P<0.001$ versus PD mice receiving control empty vector (EV) treatment. Scale bar $=50 \mu \mathrm{M}$.

moderate peritoneal fibrosis as evidenced by the thickening of the submesothelial layer accompanied with a significant upregulation and accumulation of collagen I and $\alpha$-SMA and deterioration of peritoneal functions (Figure $4 \mathrm{~b}-\mathrm{e}$ and Figure $5 \mathrm{a}-\mathrm{d}$ ). EMT was also evident at day 14 as shown by partial loss of E-cadherin whereas increasing $\alpha$-SMA expression and the appearance of cytokeratin $+\alpha$-SMA + cells in the thickened peritoneum (Figure $5 \mathrm{e}-\mathrm{g}$ ). These changes worsened by day 28 , but were attenuated by the treatment with miR-29b over day 14 to 28 (Figures 4 and 5). Of note, miR-29b treatment from day 14 onward was able to halt the progression of peritoneal fibrosis, but did not reverse the severity of peritoneal injury when compared with the disease parameters at day 14 before treatment (Figures 4 and 5). Interestingly, while pre-treatment of miR-29b significantly inhibited peritoneal angiogenesis and peritoneal dysfunctions (Figures 1 and 3), delayed treatment with miR-29b at day 14 after PD produced only a partially inhibitory effect on peritoneal angiogenesis and peritoneal functional injury (Figures 4 and 6).

\section{Blockade of the Sp1-TGF- $\beta /$ Smad Signaling is a Mechanism by which miR-29b Inhibits Peritoneal Fibrosis}

Finally, we explored potential mechanisms whereby miR-29b inhibits peritoneal fibrosis. Immunohistochemical and real- 
a

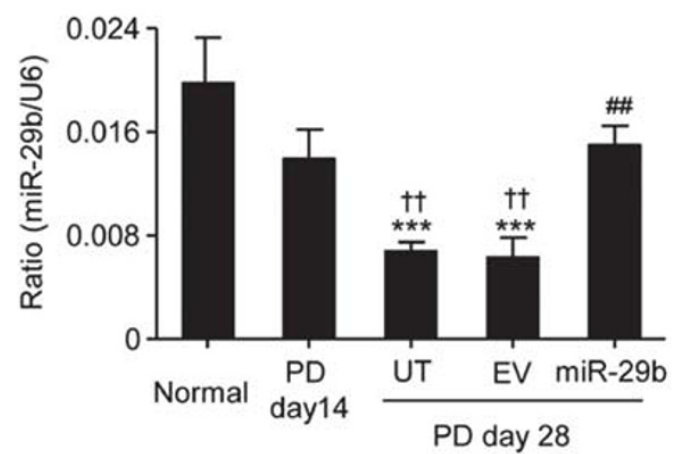

b

Peritoneal thickness

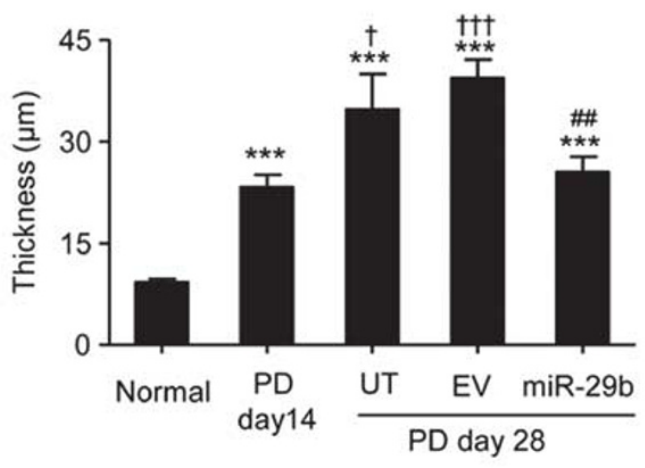

c

Masson's trichrome staining

PD day28

Normal

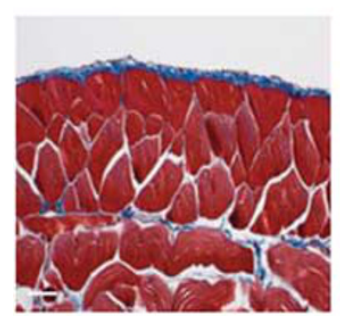

d

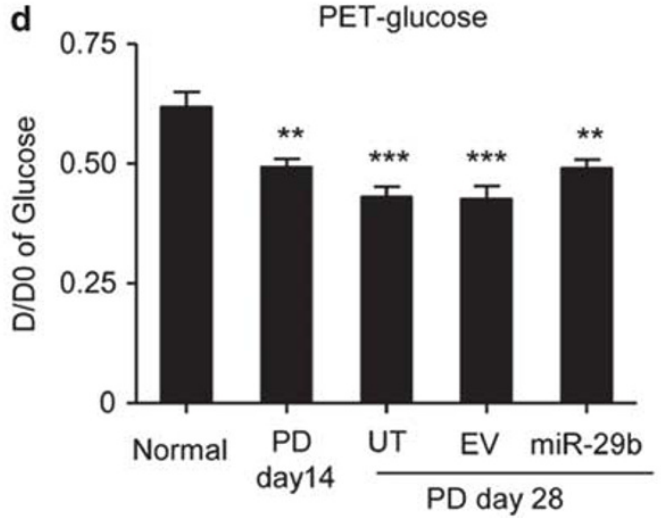

PD day14

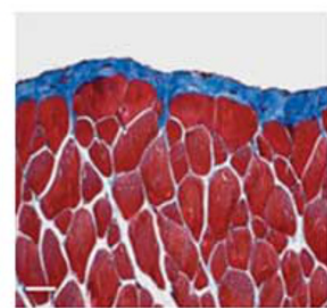

PET-glucose

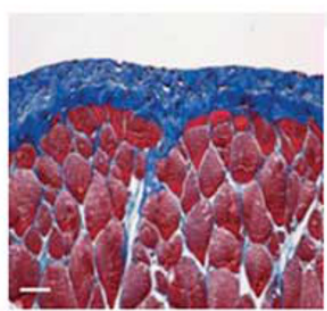

e
EV

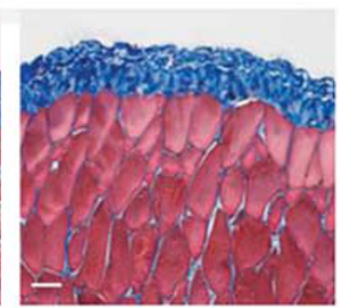

PET-BUN

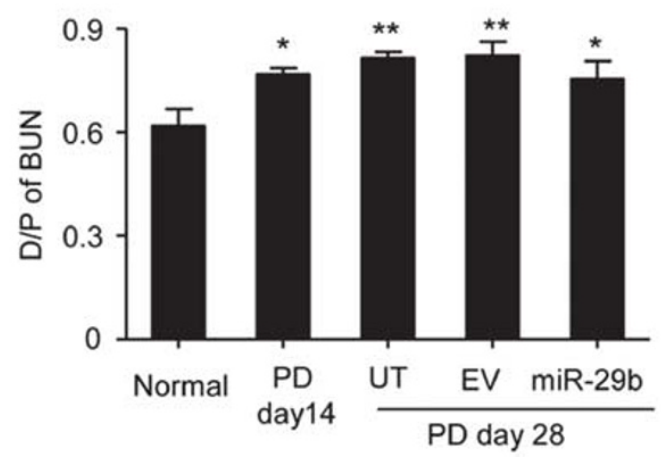

Figure 4 Delayed miR-29b treatment in the established mouse PD model halts progressive peritoneal fibrosis with a partial improvement of peritoneal functions. (a) Real-time analysis of miR-29b expression in the peritoneal tissues exposed to PDF at day 14 before miR-29b treatment and at day 28 treated with or without (UT) miR-29b or control empty vectors (EV). (b) Thickness of the peritoneal membrane. (c) Masson's trichrome staining of anterior abdominal walls. (d and e) PET-glucose and PET-BUN analysis of peritoneal function. Each bar represents mean \pm s.e.m. for at least five mice. ${ }^{*} P<0.05$, ${ }^{*} P<0.01,{ }^{* * *} P<0.001$ versus normal controls; ${ }^{\dagger} P<0.05$, ${ }^{\dagger \dagger} P<0.001$ versus PDF-treated mice at day $14 ;{ }^{\# \#} P<0.01$ versus EV control. Scale bar $=50 \mu \mathrm{M}$.

Figure 5 Delayed miR-29b treatment in the established mouse PD model halts progressive peritoneal fibrosis as demonstrated by inhibition of collagen I deposition, $\alpha$-SMA + myofibroblasts accumulation, and EMT. (a) Immunostaining of collagen I and $\alpha$-SMA + myofibroblasts accumulation in the submesothelial area of the anterior abdominal wall. (b) Western blot and real-time PCR analysis of peritoneal collagen I expression. (c and d) Realtime PCR analysis of peritoneal collagen I and $\alpha$-SMA mRNA expression. (e) Two-color immunofluorescence of anterior abdominal wall sections. Note that EMT cells (arrowheads) are identified in the fibrotic peritoneal tissues before treatment at day 14 and in both untreated (UT) and control empty vector (EV)-treated PD tissues at day 28 by being dual positive for cytokeratin (green) and $\alpha$-SMA (red), which is further illustrated in the inserted picture. Treatment of miR-29b largely blocked EMT. (f and $\mathbf{g}$ ) Western blot analysis of peritoneal E-Cadherin and $\alpha$-SMA expression. Each bar represents mean \pm s.e.m. for at least five mice. ${ }^{*} P<0.05,{ }^{*} P<0.01,{ }^{* * *} P<0.001$ versus normal controls; ${ }^{\dagger} P<0.05,{ }^{\dagger \dagger} P<0.01$ versus PDF-treated mice at day 14 ; ${ }^{\#} P<0.05,{ }^{\# \#} P<0.01,{ }^{\# \# \# P}<0.001$ versus PD mice receiving control EV. Scale bar $=50 \mu \mathrm{M}$. 

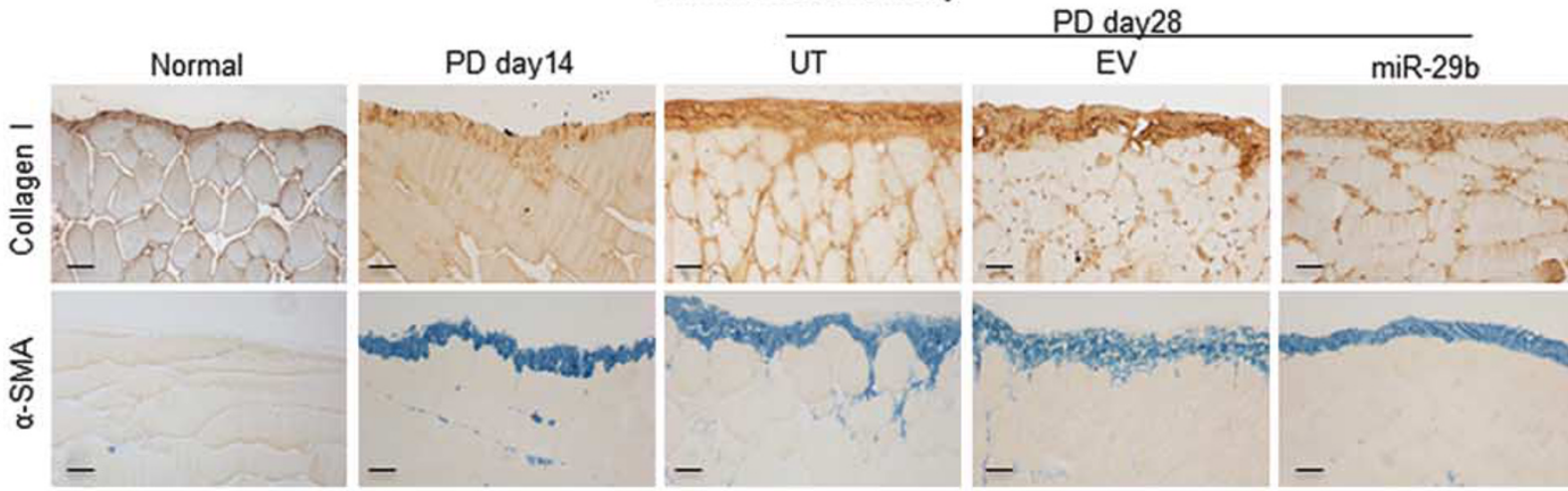

b

Collagen I protein
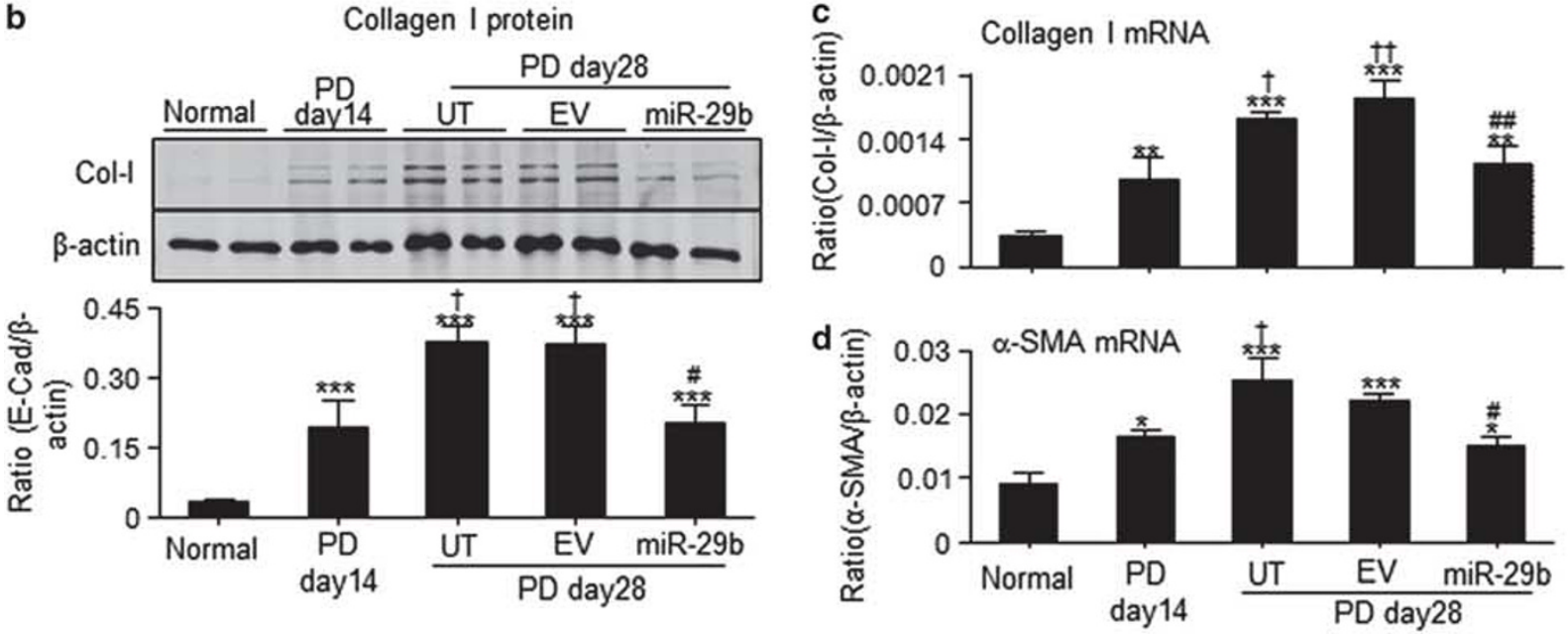

e
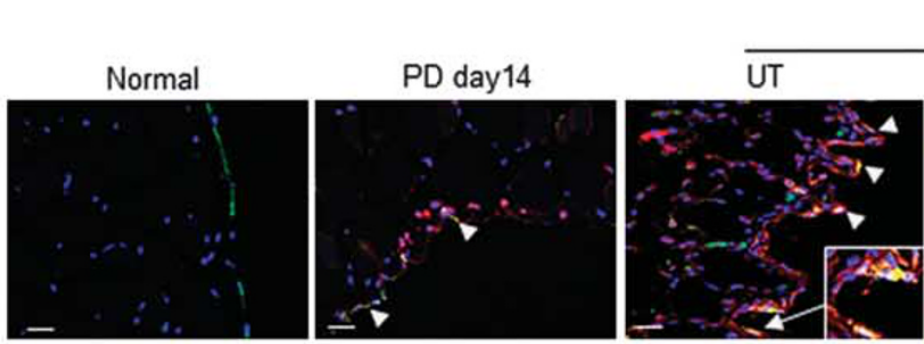

f

E-Cadherin protein

g

PD day28

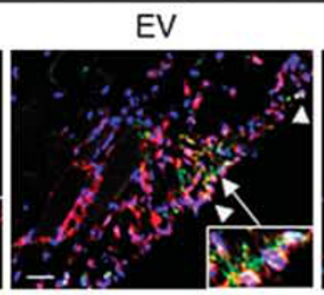

miR-29b
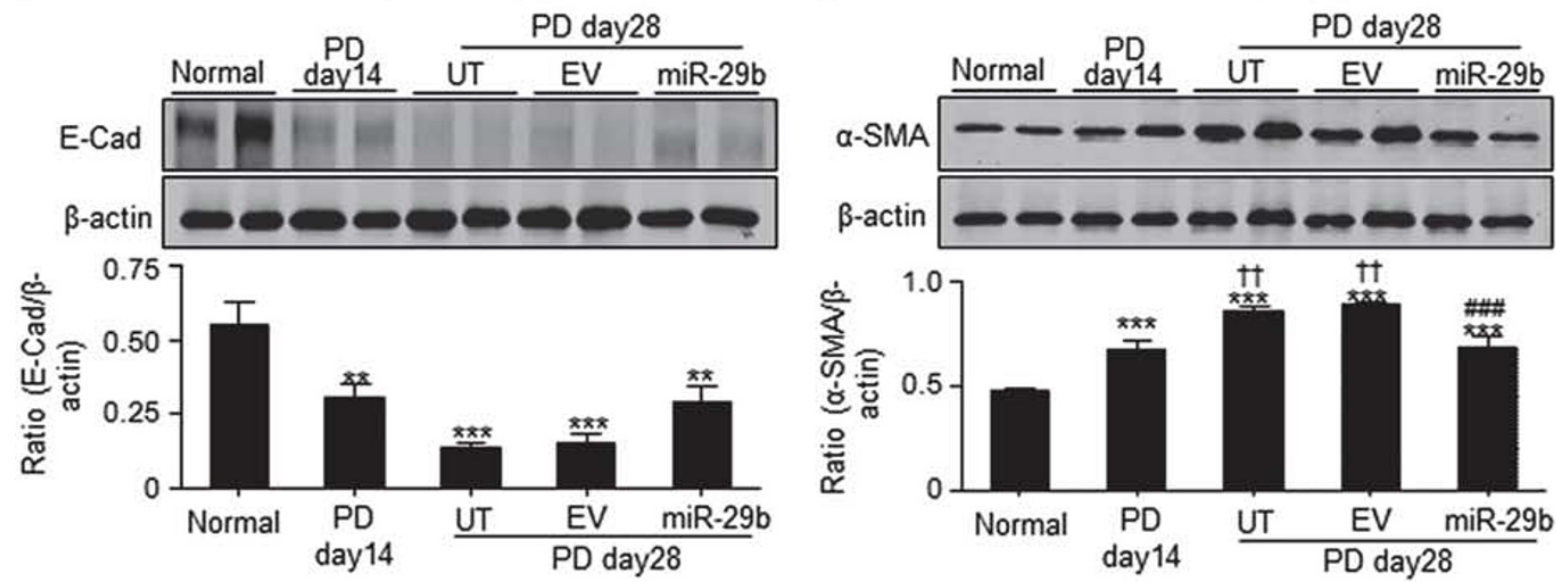
time PCR analysis revealed that loss of miR-29b in fibrotic peritoneal tissues was associated with a marked activation of TGF- $\beta /$ Smad signaling identified by a numerous phosphorylated Smad2/3-positive cells and a marked upregulation of TGF- $\beta 1$ within the thickened peritoneum (Figures 7 and 8, $a-d)$. In contrast, gene transfer with miR-29b before or after the established PD resulted in a significant inhibition of TGF- $\beta 1$ expression and activation of $\operatorname{Smad} 2 / 3$ in the peritoneal tissues (Figures 7 and 8 , a-d).

It is known that a transcription factor specificity protein 1 (Sp1) plays an important role in TGF- $\beta$-induced collagen synthesis and is a putative target of miR-29b. ${ }^{27,28}$ Thus, we further tested a hypothesis that miR-29b may inhibit peritoneal fibrosis via a mechanism of the Sp1-TGF- $\beta$ / Smad pathway. Western blot analysis revealed that expression of Sp1 was significantly upregulated in the fibrotic peritoneal tissues after PD (Figures 7 and 8, e,f). In contrast, treatment with miR-29b before or after PD produced an inhibitory effect on expression of Sp1 (Figures 7 and $8, e, f)$.

\section{DISCUSSION}

The present study identified that PD-related peritoneal fibrosis was associated with a loss of miR-29b, whereas overexpression of miR-29b before or after PD was capable of preventing or intervening progressive peritoneal fibrosis and functional injury. Results from this study suggested that miR29b may play a protective role and has therapeutic potential for PD-associated peritoneal fibrosis.

Consistent with the previous finding that miR-29 is a key player in a variety of disease models associated with fibrosis, including postmyocardial and hypertensive remodeling, ${ }^{16,17}$

\begin{tabular}{ccc} 
& PD day28 \\
\hline UT & EV & miR-29b
\end{tabular}

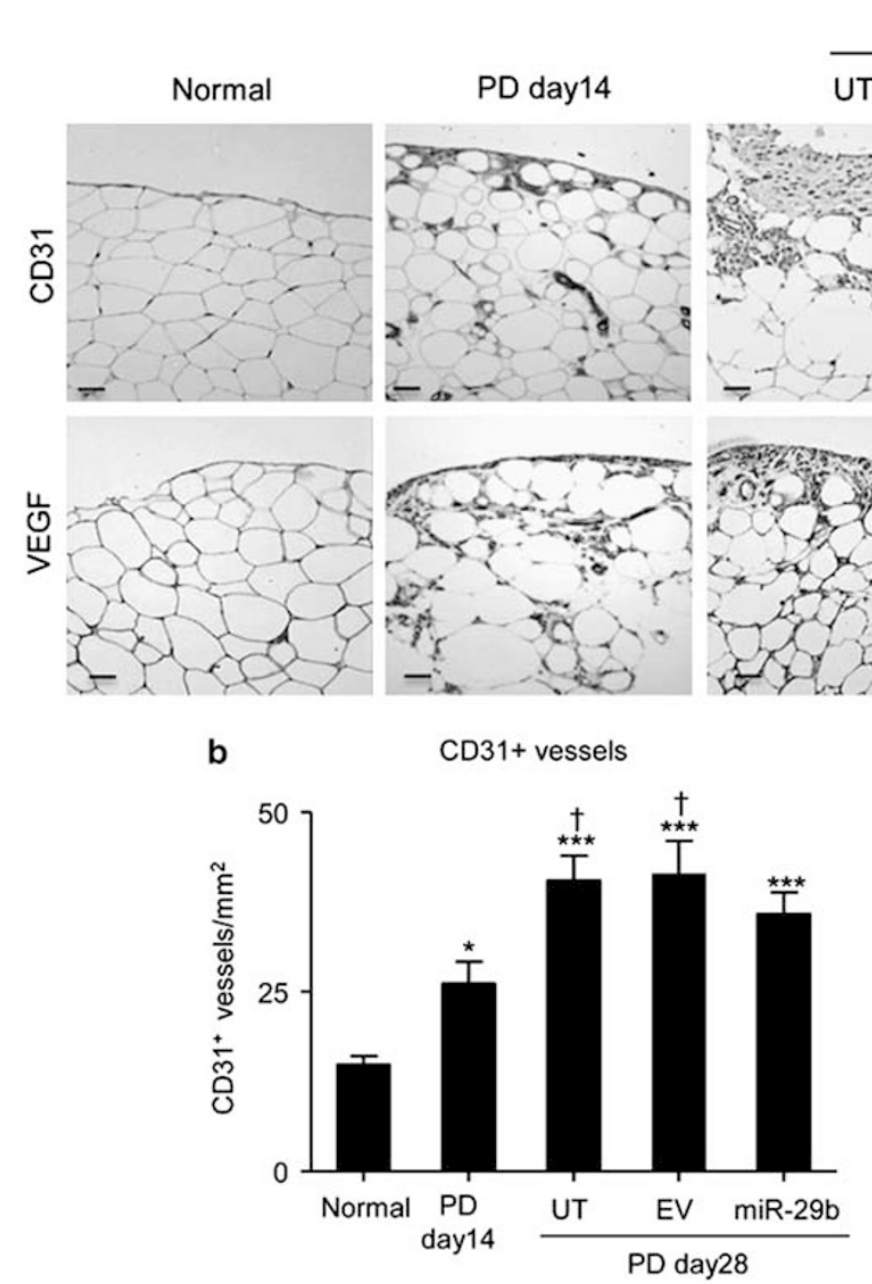

b c
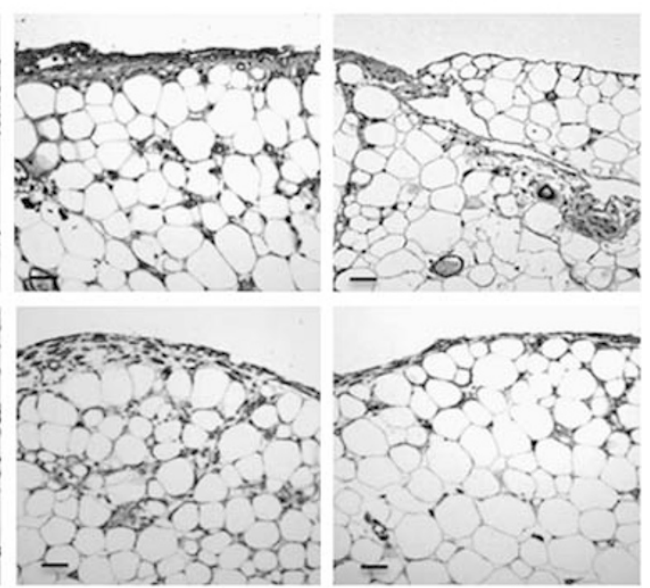
VEGF+ cells

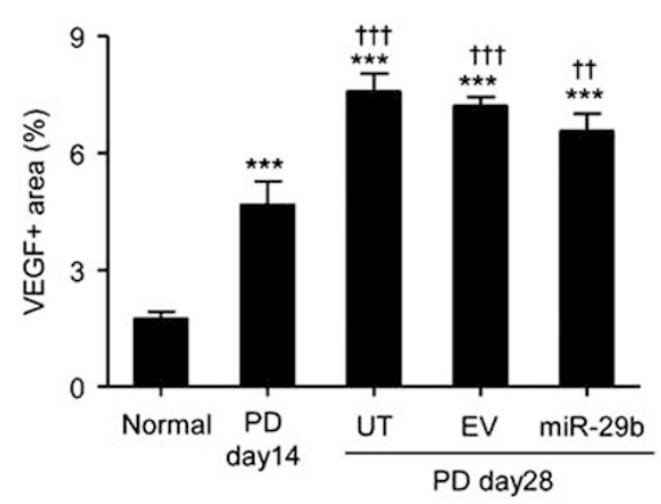

Figure 6 Effect of delayed miR-29b treatment on peritoneal angiogenesis in an established mouse model of PD. (a) Immunostaining of CD31 + vessels and VEGF + cells in the omentum with peritoneal fibrosis. (b) Quantitative analysis of CD31-positive blood vessels. (c) Quantitative analysis of VEGF expression. Each bar represents mean \pm s.e.m. for at least five mice. ${ }^{* * *} P<0.001$ versus normal controls; ${ }^{\dagger} P<0.05,{ }^{\dagger} P<0.01,{ }^{\dagger \dagger} P<0.01,{ }^{\dagger \dagger} P<0.001$ versus PDF-treated mice at day 14. UT, untreated PD mice; EV, empty vector control. Scale bar $=50 \mu \mathrm{M}$. 


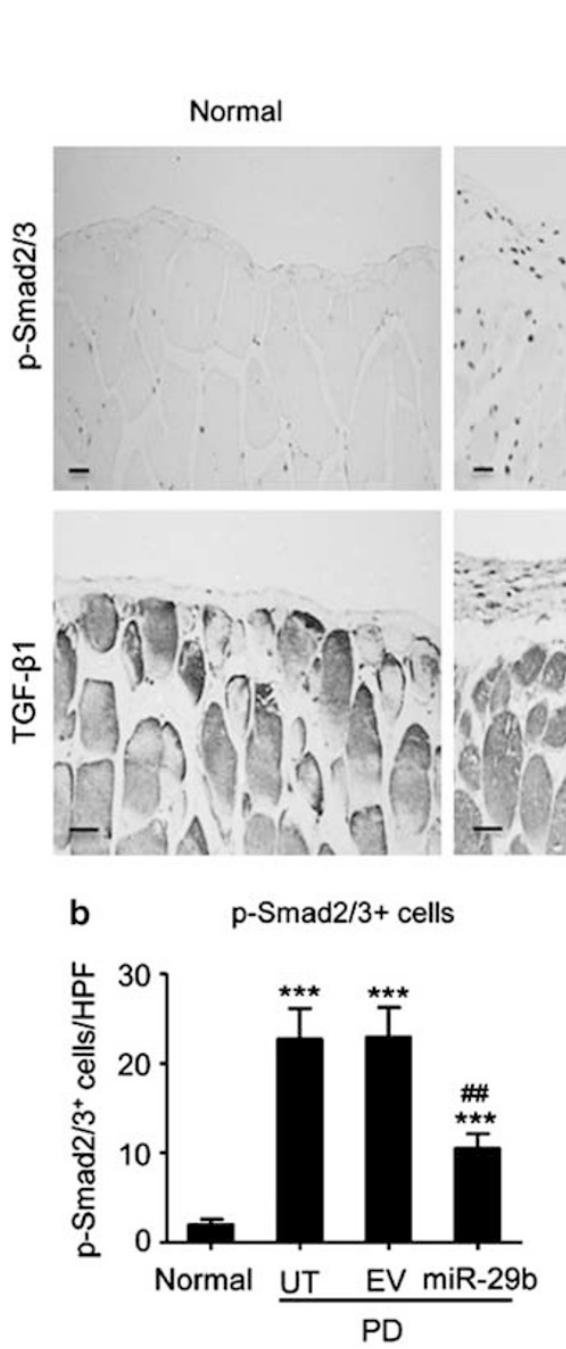

\begin{tabular}{lll}
\multicolumn{3}{c}{ PD } \\
\hline UT & EV
\end{tabular}

e

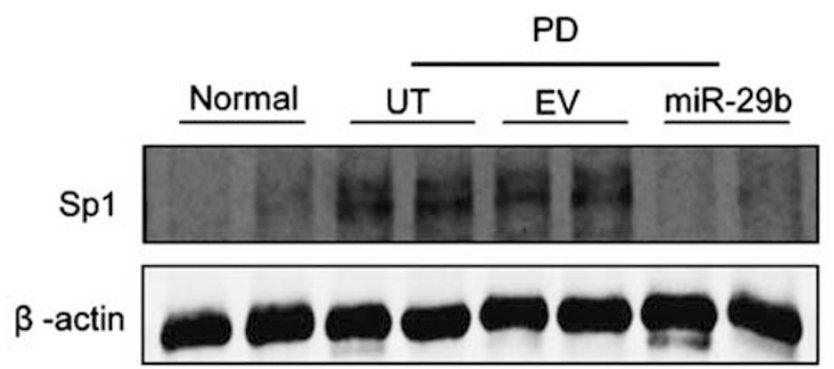

c

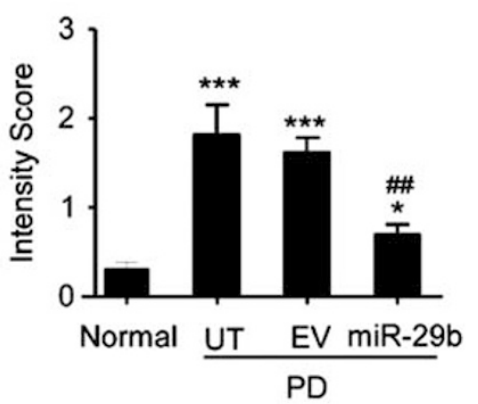

d

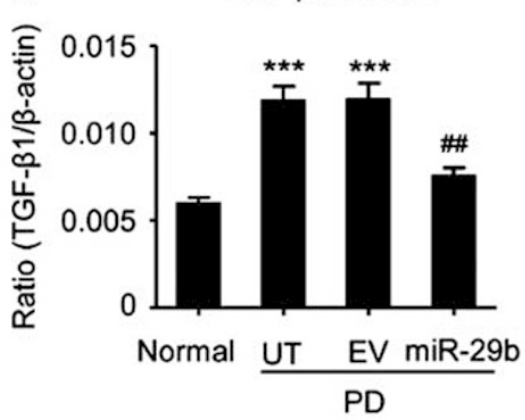

TGF-B1 mRNA
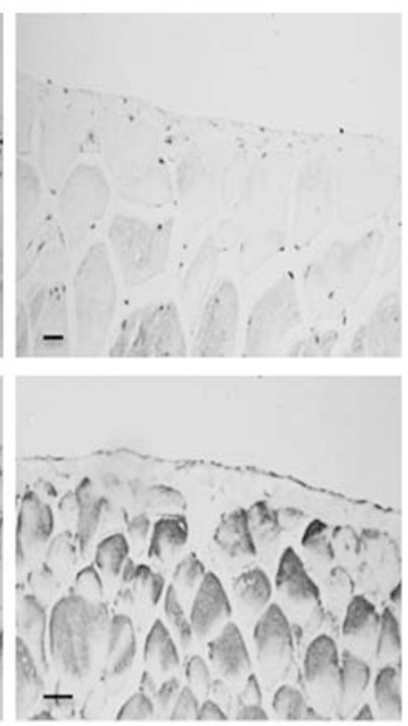

f

Sp1 quantitation

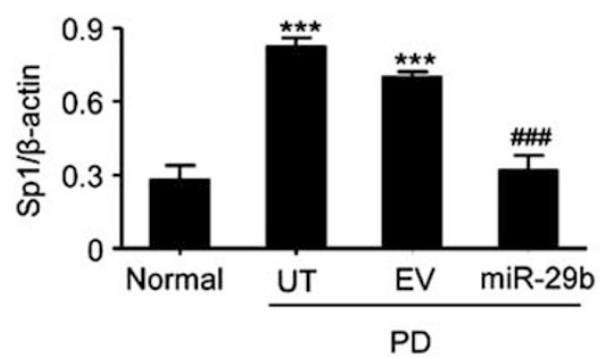

Figure 7 Pre-treatment of miR-29b inhibits activation of TGF- $\beta /$ Smad signaling and expressions of Sp1 in a mouse model of PD. (a) Immunohistochemical staining with the anti-phospho-Smad2/3 and TGF- $\beta 1$ antibodies. (b) Quantitation of phosphor-Smad2/3 in the peritoneal tissue. (c) Semi-quantitation of TGF- $\beta 1$ in the peritoneal tissue. (d) Real-time PCR analysis of peritoneal TGF- $\beta 1$ mRNA expression. (e) Western blot analysis of Sp1 expression in the peritoneal tissue. (f) Quantitative analysis of peritoneal SP1 expression by western blotting. Each bar represents mean \pm s.e.m. for at least five mice. ${ }^{*} P<0.05,{ }^{* * *} P<0.001$ versus normal controls; ${ }^{\# \#} P<0.01,{ }^{\# \# \#} P<0.001$ versus $\mathrm{PD}$ mice receiving control empty vectors (EV). Scale bar $=50 \mu \mathrm{M}$.

liver cirrosis, ${ }^{18}$ pulmonary fibrosis, ${ }^{19,20}$ and obstructive and diabetic nephropathy, ${ }^{21-23}$ the present study added new evidence for a protective and therapeutic role of miR-29b in
PD-related peritoneal fibrosis. This was supported by the finding that PDF-induced peritoneal fibrosis was associated with a loss of peritoneal miR-29b, whereas, restored 

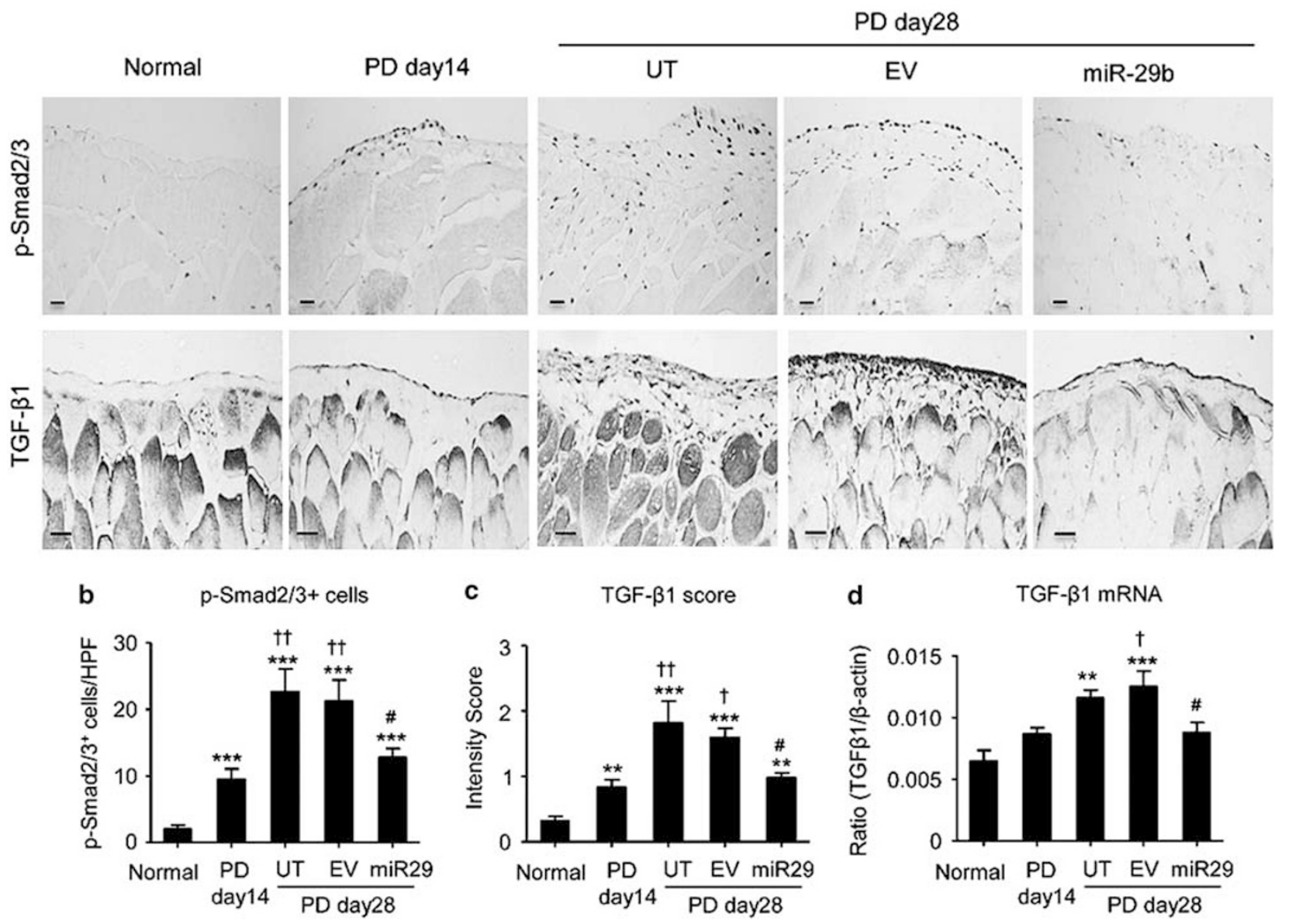

e

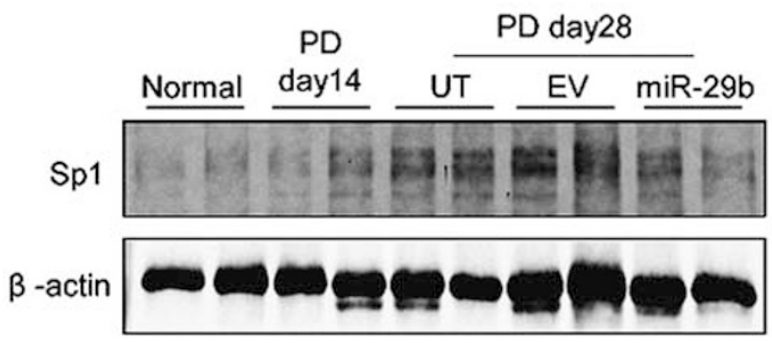

$\mathbf{f}$

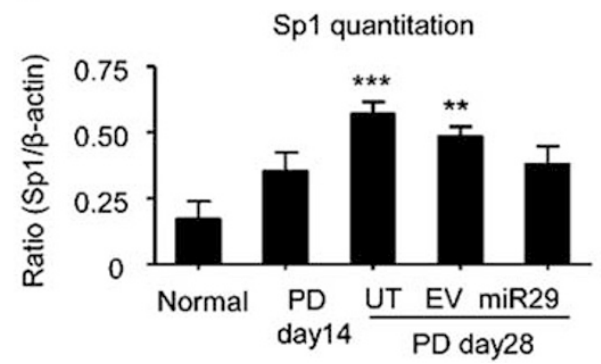

Figure 8 Effect of delayed treatment with miR-29b on activation of TGF- $\beta /$ Smad signaling and Sp1 expression in an established mouse model of PD. (a) Immunohistochemical staining with the anti-phospho-Smad2/3 and TGF- $\beta 1$ antibodies. (b) Quantitation of phosphor-Smad2/3 in the peritoneal tissue. (c) Semi-quantitation of TGF- $\beta 1$ in the peritoneal tissue. (d) Real-time PCR analysis of peritoneal TGF- $\beta 1$ mRNA expression. (e) Western blot analysis of Sp1 expression in the peritoneal tissue. (f) Quantitative analysis of peritoneal SP1 expression by western blotting. Each bar represents mean \pm s.e.m. for at least five mice. ${ }^{* *} P<0.01,{ }^{* * *} P<0.001$ versus normal controls; ${ }^{\dagger} P<0.05,{ }^{\dagger \dagger} P<0.01$ versus PDF-treated mice at day $14 ;{ }^{\#} P<0.05$ versus $P D$ mice receiving control empty vectors (EV). Scale bar $=50 \mu \mathrm{M}$.

peritoneal miR-29b protected against PD-induced peritoneal fibrosis and functional impairment, revealing a protective role of miR-29b in peritoneal injury. Furthermore, inhibition of progressive peritoneal fibrosis and functional injury in the established peritoneal disease by miR-29b demonstrated a therapeutic potential of miR-29b for peritoneal fibrosis.
In the present study, we also demonstrated that blockade of the Sp1-TGF- $\beta /$ Smad pathway may be a mechanism by which miR-29b attenuated peritoneal fibrosis. In peritoneal tissues with severe fibrosis, loss of miR-29b was associated with upregulation of Sp1 and TGF- $\beta 1$ and activation of Smad2/3, suggesting the link between miR-29 and Sp1-TGF- 
$\beta /$ Smad signaling in the pathogenesis of peritoneal fibrosis. We have previously shown that Smad3 binds the promoter of miR-29b to negatively regulate miR-29b expression in response to TGF- $\beta 1$ during renal fibrosis. ${ }^{22}$ It is also possible that a marked activation of TGF- $\beta / \mathrm{Smad} 3$ signaling in the fibrotic peritoneal tissues may downregulate miR-29b, resulting in progressive peritoneal fibrosis. There were several mechanisms whereby miR-29b may inhibit peritoneal fibrosis. First, it is well established that miR-29 suppresses fibrogenesis by directly targeting the 3'UTRs of COL1A1, COL1A2, COL3A1, ELN, and FBN1. ${ }^{16}$ Therefore, overexpression of miR-29b in the peritoneal tissue may repress the expression of collagen matrix directly. Second, miR-29 may exert its anti-fibrosis effect by inhibiting TGF- $\beta 1$ transcription. ${ }^{29}$ A recent identification of miR-29b to directly target the coding sequence of TGF- $\beta 1$ on Exon 3 supports this mechanism. ${ }^{17}$ It is now well established that TGF- $\beta$ / Smad signaling plays a functional role in the pathogenesis of peritoneal fibrosis because overexpression of TGF- $\beta 1$ in the peritoneum induced $\mathrm{EMT}^{8}{ }^{8}$ whereas inhibition of this pathway by overexpressing an inhibitor Smad7 is capable of attenuating peritoneal fibrosis in rat models of PD. ${ }^{24,30}$ Thus, blockade of TGF- $\beta /$ Smad-mediated peritoneal fibrosis may be a central mechanism by which miR-29b inhibited peritoneal fibrosis including EMT. In addition, as a putative target of miR-29, ${ }^{28} \mathrm{Sp} 1$ has been implicated in TGF- $\beta 1$ induced collagen synthesis and the interaction between Sp1 and Smad3 has been shown to enhance the TGF- $\beta$-induced fibrotic response. ${ }^{27,31}$ Therefore, blockade of the Sp1-Smad3 interaction may be another mechanism accounting for the ability of overexpressing miR-29b to inhibit TGF- $\beta /$ Smad3mediated peritoneal fibrosis.

Inhibition of peritoneal angiogenesis may also contribute to the inhibitory effect of miR-29b on peritoneal fibrosis and peritoneal dysfunction. In patients with $\mathrm{PD}$, a significant correlation between increased peritoneal vascular density and peritoneal fibrosis has been noticed and increased expression of VEGF has been reported in both dialysis solutions and peritoneal tissues. ${ }^{32,33}$ Consistent with these findings, the present study also showed that expression of peritoneal VEGF and CD31 + vessels were largely increased in response to chronic infusion of PDF, which was associated with progressive peritoneal fibrosis and deterioration of peritoneal functions. Treatment with miR-29b before PD produced a significant inhibitory effect on peritoneal angiogenesis and improvement of peritoneal function; however, delayed miR-29b treatment at day 14 after PD produced a less inhibitory effect on angiogenesis, which may explain a notion that peritoneal functional injury was not significantly improved when miR-29b treatment was delayed. Although mechanisms whereby miR-29b inhibited angiogenesis remain largely unknown, it is likely that miR-29b may act by blocking TGF- $\beta /$ Smad signaling to suppress angiogenesis because TGF- $\beta 1$ is a well-known angiogenesis factor capable of promoting VEGF expression and angiogenesis. ${ }^{7,34}$ In addition, as EMT is responsible for high solute transport rate in PD patients, ${ }^{35}$ inhibition of EMT may also be a mechanism by which miR-29b treatment improves peritoneal dysfunctions.

The present study also demonstrated that the non-invasive ultrasound-microbubble technique may be a convenient and effective method for miRNA therapy in peritoneal diseases. We have previously shown that the ultrasound-microbubble technique is able to deliver a Dox-inducible Smad7 to block TGF- $\beta /$ Smad-mediated peritoneal fibrosis. ${ }^{24,30}$ In the present study, we provided further evidence that this non-invasive ultrasound-based technique was also an effective method to deliver miRNA plasmids into the peritoneal tissues. By using this technique, we were able to effectively but non-selectively transfect the pre-miR-29b into the peritoneal tissues, thereby inhibiting peritoneal fibrosis induced by PD. Although it remains largely unclear, the mechanism by which ultrasoundmediated miR-29b gene transfer may largely be attributed to the local ultrasound-mediated miR-29b-bearing microbubble cavitation. Indeed, the cavitation can also cause a transient formation of small holes $(<5 \mathrm{~mm})$ on the cell surface, ${ }^{36}$ which may largely enhance the released gene entering into the peritoneal cells, resulting in inhibition of peritoneal fibrosis.

In conclusion, the present study identifies that after longterm exposure to PDF, peritoneal fibrosis occurs with a loss of miR-29b. In contrast, overexpression of miR-29b is capable of inhibiting PD-associated peritoneal fibrosis and improving peritoneal dysfunction. Blockade of the Sp1-TGF- $\beta /$ Smad pathway may be a mechanism by which overexpression of miR-29b inhibits peritoneal fibrosis. Results from this study suggest that treatment with miR-29b may represent as a novel and effective therapy for PD-related peritoneal fibrosis.

\section{ACKNOWLEDGMENTS}

This work was supported by grants from the Research Grants Council of Hong Kong (CUHK05/CRF/09), the Focused Investment Scheme A and B from Chinese University of Hong Kong, and the Key Program of the National Natural Science Foundation of China (Grant No.81130012).

\section{DISCLOSURE/CONFLICT OF INTEREST}

The authors declare no conflict of interest.

1. Williams JD, Craig KJ, Topley N, et al. Morphologic changes in the peritoneal membrane of patients with renal disease. J Am Soc Nephrol 2002;13:470-479.

2. Mateijsen MA, van der Wal AC, Hendriks PM, et al. Vascular and interstitial changes in the peritoneum of CAPD patients with peritoneal sclerosis. Perit Dial Int 1999;19:517-525.

3. Margetts PJ, Gyorffy S, Kolb M, et al. Antiangiogenic and antifibrotic gene therapy in a chronic infusion model of peritoneal dialysis in rats. J Am Soc Nephrol 2002;13:721-728.

4. Nishino T, Miyazaki M, Abe K, et al. Antisense oligonucleotides against collagen-binding stress protein HSP47 suppress peritoneal fibrosis in rats. Kidney Int 2003;64:887-896.

5. Tanabe K, Maeshima Y, Ichinose K, et al. Endostatin peptide, an inhibitor of angiogenesis, prevents the progression of peritoneal sclerosis in a mouse experimental model. Kidney Int 2007;71:227-238.

6. Lin CY, Chen WP, Yang LY, et al. Persistent transforming growth factorbeta 1 expression may predict peritoneal fibrosis in CAPD patients with frequent peritonitis occurrence. Am J Nephrol 1998;18:513-519. 
7. Margetts PJ, Kolb M, Galt T, et al. Gene transfer of transforming growth factor-beta1 to the rat peritoneum: effects on membrane function. J Am Soc Nephrol 2001;12:2029-2039.

8. Margetts PJ, Bonniaud P, Liu L, et al. Transient overexpression of TGF-\{beta\}1 induces epithelial mesenchymal transition in the rodent peritoneum. J Am Soc Nephrol 2005;16:425-436.

9. Loureiro J, Aguilera A, Selgas R, et al. Blocking TGF-beta1 protects the peritoneal membrane from dialysate-induced damage. J Am Soc Nephrol 2011;22:1682-1695.

10. Lan HY. Diverse roles of TGF-beta/Smads in renal fibrosis and inflammation. Int J Biol Sci 2011;7:1056-1067.

11. Meng $X M$, Huang $X R$, Chung $A C$, et al. Smad2 protects against TGF-beta/Smad3-mediated renal fibrosis. J Am Soc Nephrol 2010;21: 1477-1487.

12. Patel $\mathrm{P}$, Sekiguchi $\mathrm{Y}, \mathrm{Oh} \mathrm{KH}$, et al. Smad3-dependent and-independent pathways are involved in peritoneal membrane injury. Kidney Int 2010;77:319-328.

13. Shull MM, Ormsby I, Kier AB, et al. Targeted disruption of the mouse transforming growth factor-beta 1 gene results in multifocal inflammatory disease. Nature 1992;359:693-699.

14. Yang X, Letterio JJ, Lechleider RJ, et al. Targeted disruption of SMAD3 results in impaired mucosal immunity and diminished $\mathrm{T}$ cell responsiveness to TGF-beta. EMBO J 1999;18:1280-1291.

15. Chung $A C, Y u$ X, Lan HY. MicroRNA and nephropathy: emerging concepts. Int J Nephrol Renovasc Dis 2013;6:169-179.

16. van Rooij E, Sutherland LB, Thatcher JE, et al. Dysregulation of microRNAs after myocardial infarction reveals a role of miR-29 in cardiac fibrosis. Proc Natl Acad Sci USA 2008;105 13027-13032.

17. Zhang $Y$, Wei LH, Huang XR, et al. miR-29b as a therapeutic agent for angiotensin II-induced cardiac fibrosis by blocking TGF- $\beta / \mathrm{Smad} 3$ pathway. Mol Ther 2014;22:974-985, in press.

18. Roderburg C, Urban GW, Bettermann K, et al. Micro-RNA profiling reveals a role for miR-29 in human and murine liver fibrosis. Hepatology 2011;53:209-218.

19. Cushing L, Kuang PP, Qian J, et al. miR-29 is a major regulator of genes associated with pulmonary fibrosis. Am J Respir Cell Mol Biol 2011;45:287-294.

20. Xiao J, Meng XM, Huang XR, et al. miR-29 inhibits bleomycin-induced pulmonary fibrosis in mice. Mol Ther 2012;20:1251-1260.

21. Chen HY, Zhong X, Huang XR, et al. MicroRNA-29b inhibits diabetic nephropathy in $\mathrm{db} / \mathrm{db}$ mice. Mol Ther 2013;22:842-853.

22. Qin W, Chung AC, Huang XR, et al. TGF-beta/Smad3 signaling promotes renal fibrosis by inhibiting miR-29. J Am Soc Nephrol 2011; 22:1462-1474.
23. Wang B, Komers $\mathrm{R}$, Carew $\mathrm{R}$, et al. Suppression of microRNA-29 expression by TGF-beta1 promotes collagen expression and renal fibrosis. J Am Soc Nephrol 2012;23:252-265.

24. Nie J, Dou X, Hao W, et al. Smad7 gene transfer inhibits peritoneal fibrosis. Kidney Int 2007;72:1336-1344.

25. Zhou Q, Yang M, Lan H, et al. miR-30a negatively regulates TGF-beta1induced epithelial-mesenchymal transition and peritoneal fibrosis by targeting Snai1. Am J Pathol 2013;183:808-819.

26. Lan HY, Mu W, Nikolic-Paterson DJ, et al. A novel, simple, reliable, and sensitive method for multiple immunoenzyme staining: use of microwave oven heating to block antibody crossreactivity and retrieve antigens. J Histochem Cytochem 1995;43:97-102.

27. Poncelet AC, Schnaper HW. Sp1 and Smad proteins cooperate to mediate transforming growth factor-beta 1-induced alpha 2(I) collagen expression in human glomerular mesangial cells. J Biol Chem 2001;276:6983-6992.

28. Liu S, Wu LC, Pang J, et al. Sp1/NFкB/HDAC/miR-29b regulatory network in KIT-driven myeloid leukemia. Cancer Cell 2010;17:333-347.

29. Luna C, Li G, Qiu J, et al. Cross-talk between miR-29 and transforming growth factor-betas in trabecular meshwork cells. Invest Ophthalmol Vis Sci 2011;52:3567-3572.

30. Guo H, Leung JC, Lam MF, et al. Smad7 transgene attenuates peritoneal fibrosis in uremic rats treated with peritoneal dialysis. J Am Soc Nephrol 2007;18:2689-2703.

31. Jiang $L$, Zhou $Y$, Xiong $M$, et al. Sp1 mediates microRNA-29c-regulated type I collagen production in renal tubular epithelial cells. Exp Cell Res 2013;319:2254-2265.

32. Combet S, Miyata T, Moulin P, et al. Vascular proliferation and enhanced expression of endothelial nitric oxide synthase in human peritoneum exposed to long-term peritoneal dialysis. J Am Soc Nephrol 2000;11:717-728.

33. Io $\mathrm{H}$, Hamada $\mathrm{C}$, Ro $\mathrm{Y}$, et al. Morphologic changes of peritoneum and expression of VEGF in encapsulated peritoneal sclerosis rat models. Kidney Int 2004;65:1927-1936.

34. Szeto CC, Lai KB, Chow KM, et al. Differential effects of transforming growth factor-beta on the synthesis of connective tissue growth factor and vascular endothelial growth factor by peritoneal mesothelial cell. Nephron Exp Nephrol 2005;99:e95-e104.

35. Aroeira LS, Aguilera A, Selgas R, et al. Mesenchymal conversion of mesothelial cells as a mechanism responsible for high solute transport rate in peritoneal dialysis: role of vascular endothelial growth factor. Am J Kidney Dis 2005;46:938-948.

36. Taniyama Y, Tachibana K, Hiraoka K, et al. Local delivery of plasmid DNA into rat carotid artery using ultrasound. Circulation 2002;105: 1233-1239. 